Rev. Elet. em Gestão, Educação e Tecnologia Ambiental (e-ISSN: 2236-1170)

\title{
UTILIZAÇÃO DE PLANTAS DE BAMBU NO TRATAMENTO DE ESGOTO SANITÁRIO PELO SISTEMA DE ALAGADOS CONSTRUÍDOS
}

\author{
Karina Eliane Quege ${ }^{1}$, Rogério de Araújo Almeida², Fernando Ernesto Ucker ${ }^{3}$ \\ ${ }^{1}$ Bióloga pela Pontifícia Universidade Católica de Goiás - PUC. Mestre em Engenharia do Meio Ambiente pela \\ Universidade Federal de Goiás (PPGEMA/UFG). Especialista em Tratamento e Disposição de Resíduos Sólidos e \\ Líquidos, e em Métodos e Técnicas de Ensino. karinaequege@gmail.com \\ 2 Engenheiro Agrônomo, Mestre e Doutor em Agronomia. Especialista em Tratamento e Disposição de Resíduos \\ Sólidos e Líquidos. Professor da Escola de Agronomia e Engenharia de Alimentos - EA e do Programa de Pós- \\ Graduação em Engenharia do Meio Ambiente - PPGEMA, da Universidade Federal de Goiás - UFG.
}

rogerioufg@gmail.com

${ }^{3}$ Graduado em Engenharia Ambiental pelo Centro Universitário Franciscano. Mestre em Engenharia do Meio Ambiente pela Universidade Federal de Goiás. Atualmente cursa doutorado em Agronomia pela Universidade Federal de Goiás. ferucker@gmail.com

http://dx.doi.org/10.5902/223611707440

\section{RESUMO}

Este estudo teve por objetivo avaliar a eficiência de três espécies de bambu (Guadua angustifolia, Phyllostachys aurea e Phyllostachys bambusoides) no tratamento do esgoto sanitário, num sistema de tratamento do tipo alagados construídos, de fluxo sub-superficial vertical descendente, em Goiânia, GO. Esgoto sanitário, proveniente de uma universidade, foi aplicado em caixas de fibroamianto de $1.000 \mathrm{~L}$, preenchidas com latossolo vermelho distroférrico de textura argilosa, sobre uma camada de drenagem com 0,26 m de brita \# 3. Plantaram-se as mudas de bambu em nove leitos e três permaneceram sem plantas (testemunhas). $O$ esgoto afluente foi captado na parte inicial de uma lagoa facultativa e aplicado na superfície dos leitos de tratamento, três vezes ao dia, mediante bombeamento automatizado. Utilizou-se tempo de detenção hidráulica (TDH) de 2,3 dias, durante 150 dias e TDH de 5,2 dias por 360 dias. Dentro dos leitos de tratamento o esgoto foi mantido cinco centímetros abaixo da superfície. Coletaram-se amostras do esgoto antes e após passar por cada leito de tratamento por um período de doze meses. As amostras foram submetidas a análises laboratoriais para determinação da demanda química de oxigênio, demanda bioquímica de oxigênio, teor de nitrogênio amoniacal e de fosfato. Mediu-se a taxa de evapotranspiração de cada um dos tratamentos, calcularam-se suas eficiências na remoção da carga poluidora e acompanharam-se o comportamento das plantas nos leitos. Os resultados foram submetidos aos testes $\mathrm{F}$ e de Tukey, a $5 \%$ de probabilidade. De maneira geral, a espécie $G$. angustifolia apresentou maior eficiência na remoção da carga poluidora de todos os atributos. Houve maior remoção de DBO e de fosfato para o TDH de 5,2 dias. O TDH de 2,3 dias foi melhor para a remoção de nitrogênio amoniacal.

Palavras-chave: zona de raízes, fito-remediação, evapotranspiração, afluente. 
Rev. Elet. em Gestão, Educação e Tecnologia Ambiental (e-ISSN: 2236-1170)

\begin{abstract}
This study aimed to evaluate the efficiency of three species of bamboo (Guadua angustifolia, Phyllostachys aurea and Phyllostachys bambusoides) in the sanitary sewage treatment, with a vertically downward sub-surface flow constructed wetlands, in Goiânia, GO, Brazil. Sewage from a university was applied to asbestos cement water tanks with 1,000 liters volume capacity, filled with oxisol above a $0.26 \mathrm{~m}$ drainage layer of gravel \# 3 . Bamboo seedlings were planted in nine tanks and three tanks remained without plants (controls). The wastewater was captured at the initial part of a facultative pond and applied to the surface of the treatment tanks, three times a day, using a timer controlled pump. It was used a hydraulic retention time (HRT) of 2.3 days during 150 days and a HRT of 5.2 days for 360 days. Inside the treatment tanks, the wastewater was maintained two inches below the substrate surface. For a period of twelve months sewage samples were collected before and after passing through each treatment tank. The samples were submitted to laboratory testing for determination of chemical oxygen demand, biochemical oxygen demand, ammonia nitrogen and phosphates. The evapotranspiration rate of each treatment was measured, their efficiencies in removing the sewage pollutant load were calculated and the behavior of plants in treatment tanks were observed. The results were submitted to $\mathrm{F}$ and Tukey ( $5 \%$ of probability) tests. In general, the specie $G$. angustifolia showed greater efficiency in removing the pollution load of all attributes. There was a higher removal of BOD and phosphate at HRT of 5.2 days. The HRT of 2.3 days was better on removing ammonia nitrogen.
\end{abstract}

Keywords: root zone, phyto-remediation, evapotranspiration, affluent.

\title{
1 INTRODUÇÃO
}

Os resíduos líquidos, quando lançados diretamente num corpo receptor ou no solo, geram degradação e contaminação do meio ambiente. Para evitar tais problemas ambientais, estes resíduos necessitam de técnicas de tratamento, que muitas vezes são de alto custo e não apresentam a eficiência necessária. Todavia, existem sistemas alternativos que tratam o esgoto eficientemente e com custo mais baixo, como são os sistemas de alagados construídos.

Os sistemas de alagados construídos são tratamentos promissores, pois sua implantação e manutenção são simples. Trata-se de um processo natural, que utiliza os recursos disponíveis como a vegetação da própria região, exige pouca mecanização e mão de obra especializada; é econômico, de fácil gerenciamento e pode ser incorporado à paisagem local (DINARDI et al., 2003; MAZZOLA, 2003; VALENTIM, 2003; JASPER; BIAGGIONI; LOPES, 2007; ZANELLA, 2008). Além disto, este tratamento utiliza plantas, que associam sua beleza (efeito paisagístico) com o bom desempenho na depuração do esgoto.

Segundo Zanella (2008), o processo de tratamento por este sistema recebeu no Brasil diversas denominações, como: terras úmidas, zonas de raízes, wetlands, fitorremediação, zonas úmidas, leitos cultivados, entre outros, o que pode dificultar o reconhecimento das experiências. Para este trabalho adotou-se o termo alagados construídos (do inglês constructed wetlands).

As plantas normalmente utilizadas no sistema são aquelas mais adaptadas a ambientes aquáticos, como o aguapé (planta aquática flutuante) e a taboa (planta aquática emergente). Todavia, também podem ser utilizadas algumas espécies vegetais "marginais" ou de talude, como o bambu, que além de auxiliar o tratamento do esgoto, produz biomassa de valor econômico. 
Rev. Elet. em Gestão, Educação e Tecnologia Ambiental (e-ISSN: 2236-1170)

Assim, o objetivo deste trabalho foi avaliar a eficiência de três espécies de bambu (Guadua angustifolia, Phyllostachys aurea e Phyllostachys bambusoides) no tratamento do esgoto sanitário com um sistema do tipo alagados construídos, de fluxo sub-superficial vertical descendente.

\section{MATERIAL E MÉTODOS}

O experimento foi conduzido na Estação de Pesquisas em Tratamento de Esgoto com Plantas - EPTEP, localizada no interior da Estação de Tratamento de Esgoto - ETE Samambaia, em Goiânia - Goiás, que é operada pela Saneamento de Goiás S/A - Saneago. A ETE está situada no Campus II da Universidade Federal de Goiás - UFG e realiza a captação e o tratamento unicamente do esgoto produzido no referido campus universitário.

A EPTEP é constituída por doze leitos de tratamento, de fluxo sub-superficial vertical descendente. Cada leito é constituído por um tanque de fibra de amianto, com altura de 0,73 m, capacidade volumétrica de $1.000 \mathrm{~L}$ e área superficial de $1,45 \mathrm{~m}^{2}$.

No centro da camada inferior do substrato foi posicionado um tubo de drenagem de 50 $\mathrm{mm}$ de diâmetro, para coletar o esgoto tratado e conduzí-lo para fora do tanque. Na parte externa do tubo de drenagem foi adaptada uma união soldável, um joelho de 90 e um tubo de PVC (60 $\mathrm{cm}$ de comprimento, posicionado verticalmente). Na parte central do tubo foi conectado um registro de esfera de $1 / 2$ " para coletar o esgoto tratado, e na extremidade superior um " $\mathrm{T}$ " com redução para $25 \mathrm{~mm}$ e uma mangueira (Figura 1), que se destina à drenagem do esgoto tratado e à manutenção do nível interno de esgoto dentro do módulo de tratamento, o que é determinado pelo posicionamento do " $T$ ", pelo princípio dos vasos comunicantes.

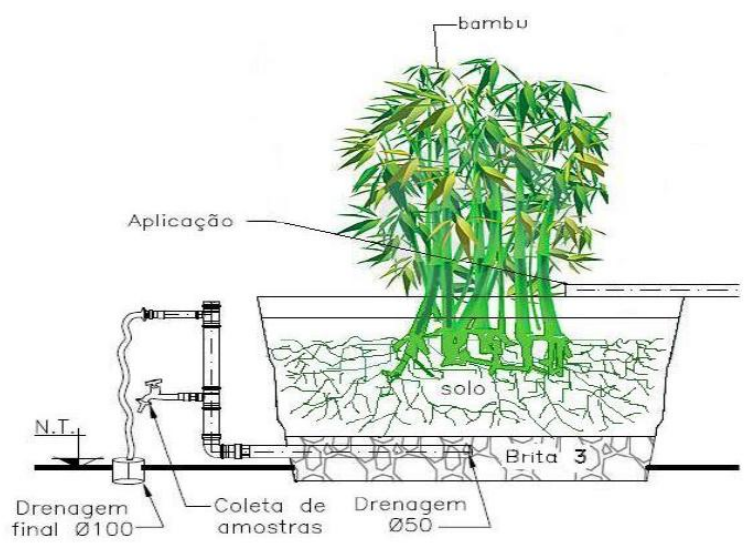

Figura 1. Vista em corte do sistema de tratamento de esgoto por alagados construídos.

Os tanques foram preenchidos, a partir do fundo, com uma camada de 0,26 m de brita \# 3, seguida por uma camada de 0,32 $\mathrm{m}$ de latossolo vermelho distroférrico de textura argilosa (OLIVEIRA JÚNIOR et al., 2005; FIGUEIREDO et al., 2009). Este solo possui baixa densidade aparente, de $0,92 \mathrm{~g}$ a $1,15 \mathrm{~g} / \mathrm{cm}^{3}$, e porosidade de alta a muito alta, $60 \%$ a $69 \%$, indicando boas condições físicas (SANTOS et al., 2007). Foram deixados $0,15 \mathrm{~m}$ de espaço livre até a borda superior de cada tanque. O espaço vazio destina-se ao acúmulo das águas de chuva e dos esgotos aplicados, para evitar transbordamentos. 
Rev. Elet. em Gestão, Educação e Tecnologia Ambiental (e-ISSN: 2236-1170)

Como volume útil foi considerado o volume de vazios do substrato do leito de tratamento, desconsiderando-se o volume ocupado pelas raízes das plantas. Os volumes de brita \# $3\left(0,309 \mathrm{~m}^{3}\right)$ e de solo $\left(0,425 \mathrm{~m}^{3}\right)$ foram determinados matematicamente mediante cálculo de volumes de troncos de cone. O percentual de vazios da brita \# 3 foi de $51 \%$, conforme observado por Abrantes (2009), cujo trabalho foi realizado na mesma estação de pesquisas. O percentual de vazios do solo (53\%) foi determinado em laboratório, utilizando-se o método do picnômetro preenchido com o solo e água até uma marca de aferição. Os volumes de vazios de brita \# $3\left(0,157 \mathrm{~m}^{3}\right)$ e de solo $\left(0,225 \mathrm{~m}^{3}\right)$ foram calculados multiplicando seus volumes por seus percentuais de vazios. $O$ volume útil do leito de tratamento $\left(0,382 \mathrm{~m}^{3}\right)$ foi calculado pela soma do volume de vazios da camada de brita \# 3 com o volume de vazios da camada de solo.

A aplicação de esgoto no sistema experimental de tratamento foi feito por bombeamento do esgoto tido como bruto (afluente) por meio de uma bomba centrífuga de rotor semiaberto, sustentada por um flutuador. A bomba foi posicionada no início da lagoa facultativa da ETE Samambaia, exatamente no alinhamento dos tubos de alimentação da lagoa, equidistante destes. $O$ esgoto bombeado foi conduzido até os tanques por meio de tubos de PVC de $25 \mathrm{~mm}$ de diâmetro e aplicado no centro superior de cada tanque, sobre a camada de terra. A taxa de aplicação foi controlada pelo tempo de funcionamento da bomba.

Após a aplicação, o esgoto se distribuía pela superfície do substrato e percolava até a camada de brita \# 3 , onde penetrava nos orifícios do tubo de drenagem e era conduzido à parte externa do tanque. $O$ esgoto drenado de todos os leitos foi conduzido por mangueiras até um tubo PVC de $100 \mathrm{~mm}$ de diâmetro. Na parte final do tubo havia um reservatório de onde o esgoto drenado era bombeado de volta à lagoa facultativa.

O nível do esgoto dentro dos leitos foi mantido cinco centímetros abaixo da superfície do substrato, mediante o posicionamento (inclinação) do tubo externo de drenagem e do " $\mathrm{T}$ ", de forma a evitar a proliferação de insetos e a liberação de maus odores.

Nove leitos de tratamento foram plantados com mudas de bambu de três espécies diferentes e três leitos permaneceram sem plantas, com o objetivo de servirem de testemunhas (quatro tratamentos e três repetições). A posição de cada tratamento (Figura 2) foi definida aleatoriamente, mediante sorteio. Em cada leito foram plantadas quatro mudas de bambu. Não foi utilizado nenhum tipo de fertilizante ou nutriente nos leitos. 
Rev. Elet. em Gestão, Educação e Tecnologia Ambiental (e-ISSN: 2236-1170)

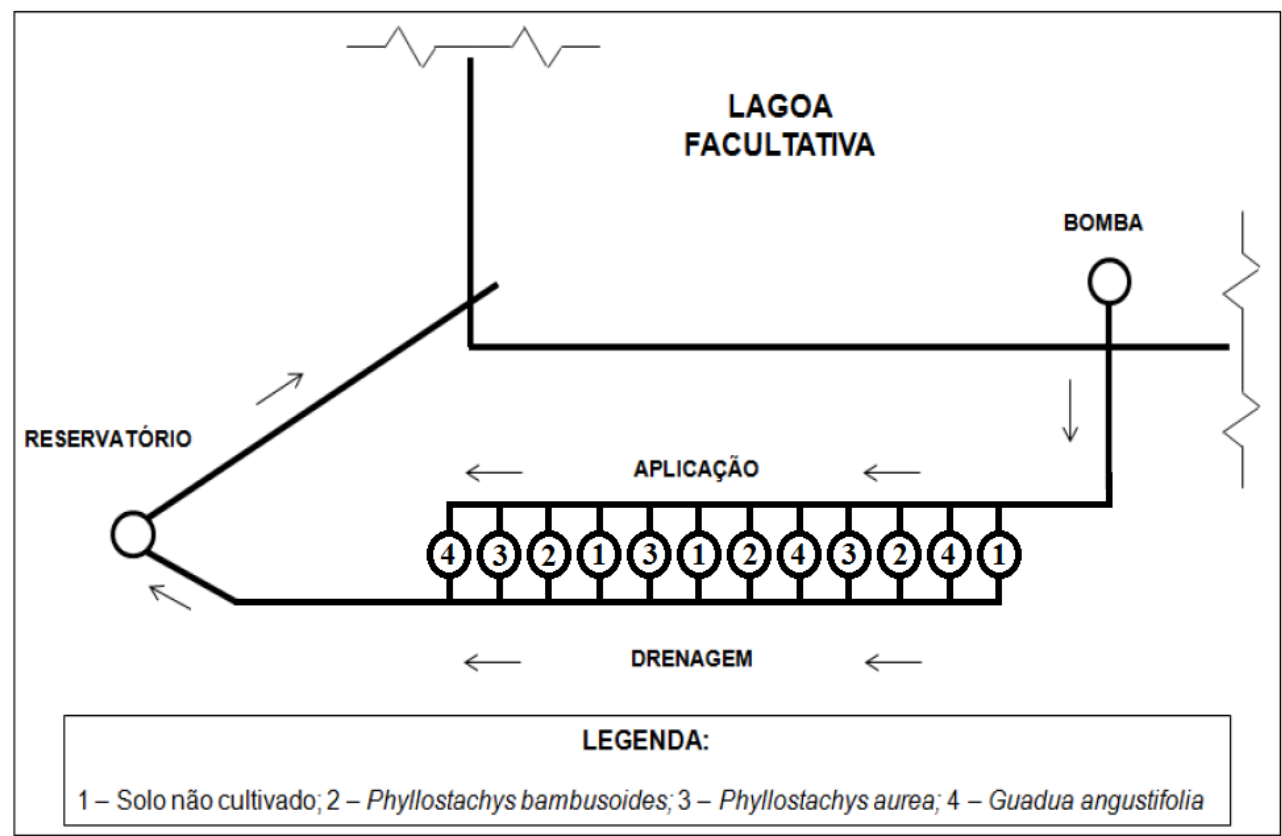

Figura 2. Disposição dos tratamentos (espécies de bambu e do solo não cultivado) no sistema experimental de tratamento de esgoto por alagados construídos.

As três espécies de bambu utilizadas foram: Guadua angustifolia, Phyllostachys aurea e Phyllostachys bambusoides. O transplantio das mudas de bambu foi realizado em 01 de fevereiro de 2010 no período vespertino, sendo irrigadas logo em seguida. A irrigação das mudas foi realizada três vezes por semana, com água de abastecimento público, sendo que em dias chuvosos não houve irrigação. A aplicação do esgoto iniciou-se no dia 30 de março de 2010, 57 dias do plantio, após desenvolvimento e o pegamento satisfatório de todas as espécies vegetais, conforme orientação de Philippi e Sezerino (2004).

Em 30 de março de 2010 iniciaram-se as aplicações de esgoto, três vezes ao dia (7h00min, $12 \mathrm{~h} 00 \mathrm{~min}$ e $17 \mathrm{~h} 00 \mathrm{~min}$ ), por períodos de 31 minutos para cada aplicação, o que resultou num tempo de detenção hidráulica (TDH) de 2,3 dias. Em 15 de setembro do mesmo ano, a duração das aplicações foi reduzida para 14 minutos, aumentando o TDH para 5,2 dias. Assim, a taxa diária inicial de aplicação de esgoto no sistema (TDH = 2,3 d) foi de aproximadamente 164 litros (113 L $\mathrm{m}^{-2} \mathrm{~d}^{-1}$ ) e a taxa final (TDH $=5,2 \mathrm{~d}$ ) foi de 74 litros $\left(51 \mathrm{~L} \mathrm{~m}^{-2} \mathrm{~d}^{-1}\right)$ subdivididas em três aplicações. As aplicações foram realizadas de segunda a sexta-feira, não sendo aplicado esgoto nos finais de semana.

Durante a execução do experimento foram coletadas amostras do esgoto antes e após passar pelos leitos de tratamento. A amostragem ocorreu nos registros de coleta de cada um dos doze leitos de tratamento, que representou o esgoto tratado (afluente), e no tubo de retorno da linha de alimentação (Figura 1), que representou o esgoto considerado bruto (afluente), totalizando treze pontos de coleta.

As amostras foram submetidas a análises laboratoriais para a determinação da demanda química de oxigênio - DQO, demanda bioquímica de oxigênio - DBO, teor de nitrogênio amoniacal e teor de fosfato. Para a realização das análises foram utilizados os métodos padronizados pelo Standard Methods for the Examination of Water and Wastewater (APHA, 1992).

Após o início da aplicação do esgoto, mensalmente, e em períodos de cinco dias consecutivos (segunda-feira a sexta-feira), foram realizadas medições das taxas de 
evapotranspiração do sistema de alagados construídos. As saídas das drenagens dos módulos de tratamento foram interligadas, por meio de mangueiras, a tambores de $200 \mathrm{~L}$ de capacidade volumétrica.

Diariamente, mediu-se o volume armazenado em cada tambor, e por meio da Eq. (1) estimou-se a taxa de evapotranspiração de cada tratamento. As medições foram realizadas nas semanas de coleta de amostras, e seus valores foram utilizados nos cálculos de eficiência do sistema.

$$
\mathrm{ETP}=\mathrm{P}+\frac{\mathrm{I}-\mathrm{D}}{\mathrm{S}}
$$

Em que:

ETP - Evapotranspiração, mm;

$P$ - Precipitação efetiva, $\mathrm{mm}$;

I - Esgoto aplicado, L;

D - Drenagem, L;

$\mathrm{S}$ - Área superficial do módulo, $\mathrm{m}^{2}$.

Após o cálculo do volume de esgoto evapotranspirado, realizou-se o cálculo da eficiência percentual do sistema de tratamento do tipo alagado construído na remoção da carga poluidora, utilizando-se a Eq. (2), conforme sugerido por Almeida e Ucker (2011):

$$
\mathrm{Ef}(\%)=\frac{(\mathrm{Ce} \times \mathrm{Ve})-(\mathrm{Cs} \times \mathrm{Vs})}{(\mathrm{Ce} \times \mathrm{Ve})} \times 100
$$

Em que:

Ef (\%) - eficiência percentual de remoção da carga contaminante de determinado atributo;

Ce - concentração do atributo na entrada do sistema;

Ve - volume de esgoto na entrada do sistema;

Cs - concentração do atributo na saída do sistema;

Vs - volume de esgoto na saída do sistema.

Os resultados das eficiências na remoção da carga poluidora foram submetidos à análise de variância (teste F) e ao teste de Tukey ( $5 \%$ de probabilidade), para comparação entre os tratamentos.

\section{RESULTADOS E DISCUSSÃO}

Os valores médios da eficiência percentual na remoção dos atributos físico-químicos no esgoto sanitário após serem submetidos ao tratamento pelo sistema de alagados construídos com plantas de bambu são apresentados na Tabela 1. 
Rev. Elet. em Gestão, Educação e Tecnologia Ambiental (e-ISSN: 2236-1170)

Tabela 1 Médias da evapotranspiração e da eficiência percentual de remoção dos atributos no sistema de tratamento de esgoto por alagados construídos.

\begin{tabular}{cccccc}
\hline Tratamento $^{1}$ & ETP $^{\mathbf{2}}$ & DBO $^{\mathbf{3}}$ & DQO $^{4}$ & Fosfato & N. Amon \\
\hline Solo & 5,2 & $89,2 \mathrm{~b}$ & $87,5 \mathrm{~b}$ & $94,5 \mathrm{a}$ & $95,3 \mathrm{a}$ \\
Bamb & 7,2 & $90,0 \mathrm{~b}$ & $83,2 \mathrm{~b}$ & $87,0 \mathrm{~b}$ & $86,3 \mathrm{~b}$ \\
Aurea & 8,1 & $89,0 \mathrm{~b}$ & $82,1 \mathrm{~b}$ & $86,4 \mathrm{~b}$ & $89,5 \mathrm{~b}$ \\
Angu & 86,0 & $98,3 \mathrm{a}$ & $97,1 \mathrm{a}$ & $98,4 \mathrm{a}$ & $98,2 \mathrm{a}$ \\
\hline
\end{tabular}

${ }^{1}$ Solo: tratamento sem a presença de planta; Bamb: Phyllostachys bambusoides; Aurea: Phyllostachys aurea; Angu: Guadua angustifolia. ${ }^{2}$ ETP: evapotranspiração (L). ${ }^{3}$ DBO: demanda bioquímica de oxigênio. ${ }^{4}$ DQO: demanda química de oxigênio. ${ }^{5} \mathrm{~N}$. Amon: nitrogênio amoniacal. Médias seguidas pela mesma letra nas colunas não diferem entre si pelo teste de Tukey a $5 \%$ de probabilidade.

\section{Demanda Bioquímica de Oxigênio}

A média percentual na remoção da demanda bioquímica de oxigênio no leito com $G$. angustifolia foi de $98,3 \%$. No leito com $P$. bambusoides foi de $90,0 \%$, com $P$. aurea foi de $89,0 \%$ e no leito não vegetado foi de $89,2 \%$ (Tabela 1 ). As eficiências dos tratamentos na remoção da DBO foram bastante variáveis até os 150 dias do início da aplicação do esgoto, mas, a partir de então, aumentaram e se aproximaram de uma média superior a $90 \%$, aos 300 dias, o que pode ser atribuído ao amadurecimento do sistema e, também, ao aumento do TDH. Conforme as plantas adaptaram-se ao sistema, a remoção da DBO cresceu (Figura 3). O tratamento com G. angustifolia mostrou-se mais eficiente que os demais, desde o início das análises.

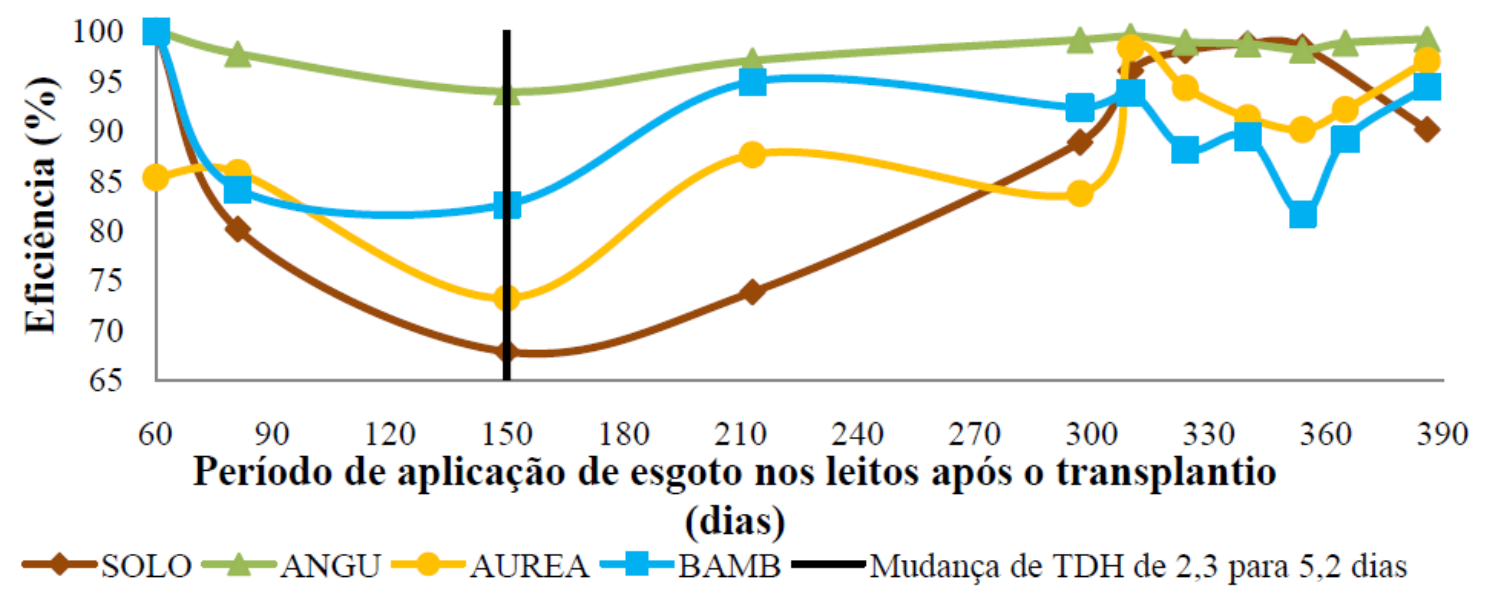

\section{Figura 3. Evolução da eficiência na remoção da DBO nos módulos de tratamento} com bambu entre 60 a 390 dias após o início da aplicação do esgoto.

Goiânia, GO. 2011.

Costa et al. (2003) ao analisar um sistema de tratamento de esgoto de fluxo sub-superficial, cultivado com taboa, obtiveram uma redução média de $88 \%$ na DBO com um tempo de detenção de 10 dias. Brix (1987), utilizando um sistema de alagados construídos na Dinamarca, observou, para uma área de 1,9 metros quadrados por habitante, cultivada com caniço, uma remoção de $66 \%$ para DBO. Tal resultado é inferior ao obtido neste estudo, onde observou-se uma remoção acima de $89 \%$, chegando a $98,3 \%$ de eficiência, para uma área de $2 \mathrm{~m}^{2}$ por habitante.

Na Tailândia, Ta-oun et al. (2003), utilizando o capim Vetiver, obtiveram resultados de aproximadamente $94 \%$ de remoção da DBO em um sistema de tratamento de esgoto por alagados 
Rev. Elet. em Gestão, Educação e Tecnologia Ambiental (e-ISSN: 2236-1170)

construídos. Utilizando esta mesma planta, Ash e Truong (2003) trataram o esgoto doméstico em Queensland, na Austrália, e verificaram uma eficiência de 95,8\% na remoção da demanda bioquímica de oxigênio.

\section{Demanda Química de Oxigênio}

A média percentual na remoção da carga da demanda química de oxigênio no leito com $G$. angustifolia foi de $97,1 \%$. No leito com $P$. bambusoides foi $83,2 \%$ e com $P$. aurea foi de $82,1 \%$ e no solo sem vegetação foi de $85,7 \%$. O G. angustifolia foi mais eficiente que os demais tratamentos, que não diferiram entre si (Tabela 1). O solo apresentou tendência de melhor eficiência (embora não significativa) de remoção da DQO comparado com as plantas do gênero Phyllostachys, com remoção de $85,7 \%$, que pode ser explicado pelo pequeno porte das plantas e, também, pela alta concentração de rizomas, que podem ter induzido a formação de caminhos preferenciais do esgoto dentro do leito e impossibilitado uma melhor degradação da matéria orgânica pelos microrganismos, pelo menor tempo de contato.

O tratamento com $G$. angustifolia mostrou-se mais eficiente que os demais desde o início do experimento. As eficiências dos demais tratamentos diminuíram até os 210 dias do início da aplicação do esgoto, crescendo até $90 \%$ aos 340 dias e voltaram a cair aos 390 dias (Figura 4).

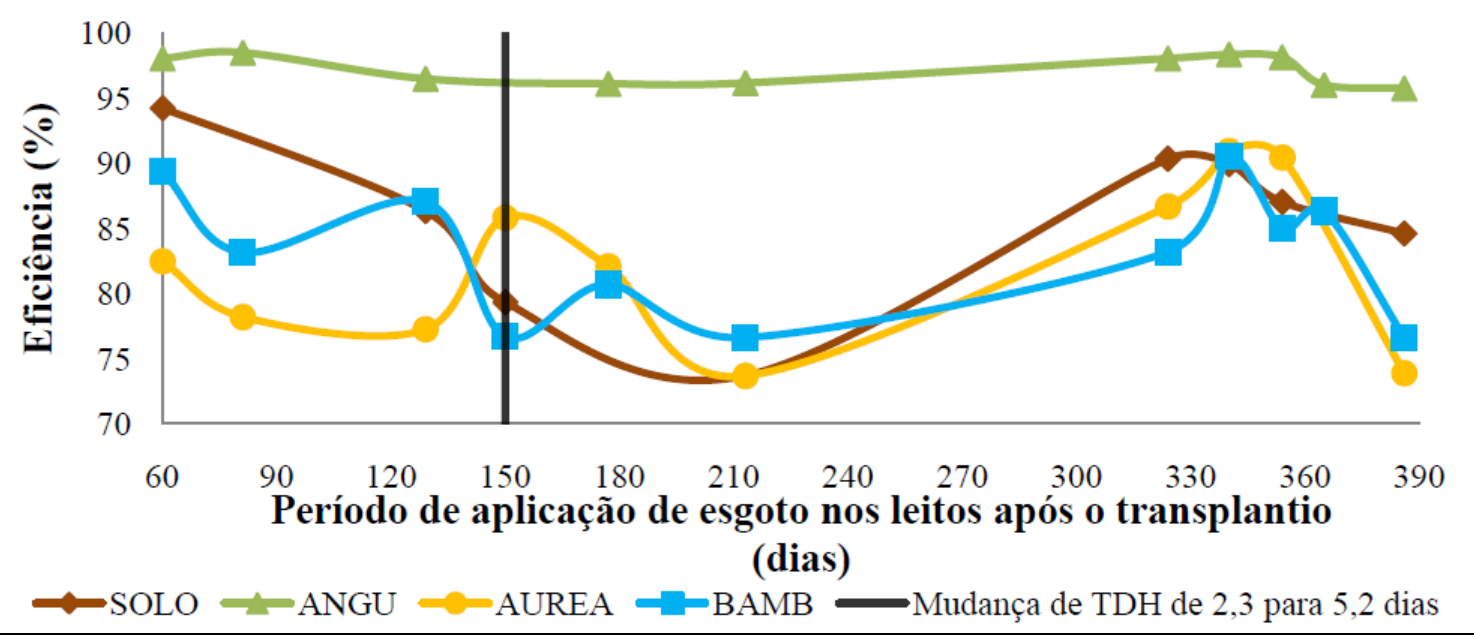

Figura 4 Evolução da eficiência na remoção da DQO nos módulos de tratamento com bambu entre 60 a 390 dias após o início da aplicação do esgoto.

Goiânia, GO. 2011.

Até 150 dias o TDH foi de 2,3 dias e a partir daí passou para 5,2 dias. A espécie $G$. angustifolia mostrou resultados mais constantes, que persistiram, mesmo com a mudança do TDH, que não afetou sua eficiência.

A redução da DQO obtida neste trabalho é similar ao encontrado por alguns autores no Estado de Goiás. Almeida, Oliveira e Kliemann (2007), ao utilizar substrato areia num sistema vegetado com Lírio do Brejo (Hedychium coronarium), obtiveram eficiência de $89,4 \%$. Abrantes (2009) obteve uma média percentual de remoção de $72,7 \%$ utilizando um fluxo sub-superficial vertical ascendente seguido de um descendente, com utilização de taboa e caniço.

Em outras regiões tem-se observado resultados também satisfatórios na redução da DQO. Haberl et al. (2007) analisaram um alagado construído de fluxo vertical plantado com caniço na região superior da Áustria e observaram remoções para DQO que variaram de $86 \%$ a 95\%. Solano, 
Rev. Elet. em Gestão, Educação e Tecnologia Ambiental (e-ISSN: 2236-1170)

Soriano e Ciria (2004) analisaram a eficiência de plantas na remoção de DQO durante um período de dois anos sendo que, no verão do primeiro ano, obtiveram uma remoção de $77 \%$ para caniço e de $76 \%$ para taboa e no verão do segundo ano, observaram remoção de $85 \%$ e $88 \%$ para as respectivas espécies vegetais.

\section{Fosfato}

A média da remoção percentual de fosfato foi de $98,4 \%$ nos leitos com $G$. angustifolia e $94,5 \%$ no solo não vegetado (Tabela 1 ). Estes dois tratamentos não diferiram entre si e foram mais eficientes que os demais, que também não diferiram entre si. $P$. bambusoides removeu $87,0 \%$ do fosfato e $P$. aurea removeu $86,4 \%$. Este comportamento (dois mais eficientes e dois menos eficientes) manteve-se desde o início da aplicação do esgoto sanitário nos leitos (Figura 5).

Segundo Moortel et al. (2009), a liberação de oxigênio pelas raízes pode aumentar a capacidade de adsorção do fósforo por meio dos substratos, o que pode ter influenciado para fazer com que os sistemas de tratamento com plantas tivessem uma eficiência de remoção maior do que os sistemas sem a presença das plantas. Meira et al. (2001) destacam que em alagados construídos, com brita e areia como substrato, os principais mecanismos para remoção de fósforo são a sedimentação e a precipitação, uma vez que tais materiais têm baixa capacidade de adsorção e a exportação do fósforo do sistema ocorre devido à absorção pela vegetação.

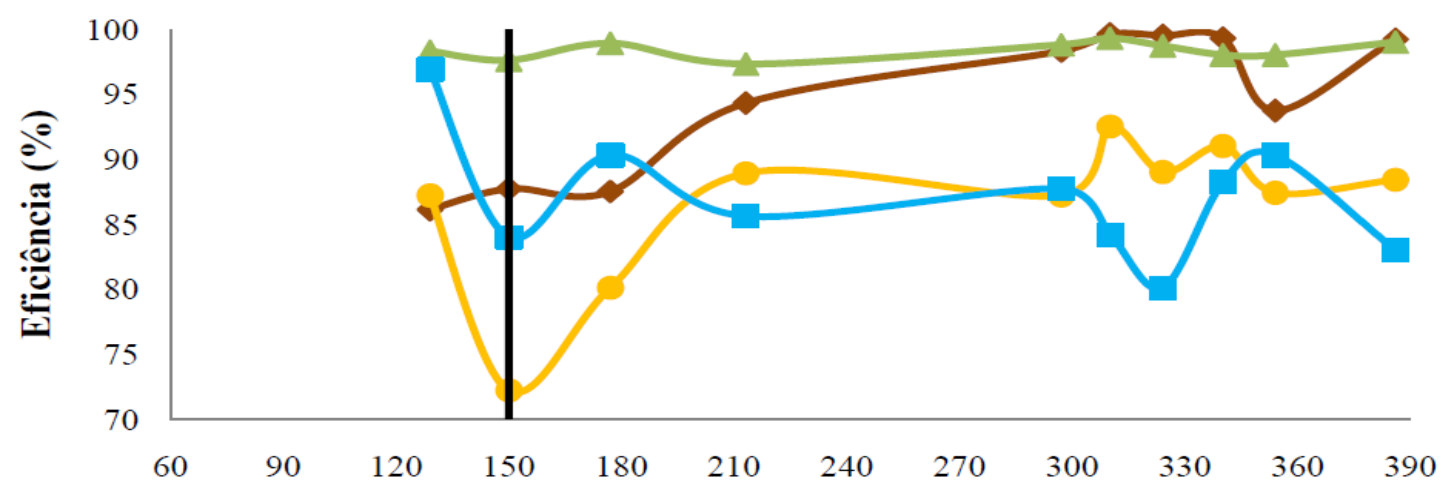

Período de aplicação de esgoto nos leitos após o transplantio

(dias)

$\multimap$ SOLO $\multimap$ ANGU $\multimap$ AUREA $\leftrightharpoons$ BAMB $\longrightarrow$ Mudança de TDH de 2,3 para 5,2 dias

Figura 5 Evolução da eficiência na remoção de fosfato nos módulos de tratamento com bambu entre 60 a 390 dias após o início da aplicação do esgoto.

Goiânia, GO. 2011.

Experimentos realizados por Brix (1987) na Dinamarca revelaram uma faixa de remoção de fósforo total de $11 \%$ a $94 \%$. Os solos caracterizados com altos teores de matéria orgânica mostraram que a adsorção de substâncias húmicas e fúlvicas na rizosfera foi extremamente importante para remoção do fósforo.

Brix, Arias e Johansen (2003) verificaram uma remoção de fósforo da ordem de $98 \%$ ao analisar um alagado construído semeado com caniço para tratar águas residuárias de uma pequena comunidade na Dinamarca. Souza e Bernardes (1996) ao utilizar leitos cultivados com taboa no pós-tratamento de esgotos domésticos, com um tempo de detenção de 2,7 dias, verificaram $40,0 \%$ de remoção de fósforo. 
Rev. Elet. em Gestão, Educação e Tecnologia Ambiental (e-ISSN: 2236-1170)

Em estudo realizado na Venezuela, Mayorca (2007) obteve percentual de remoção do atributo fósforo total de $74 \%$ em um sistema de alagados construídos para tratar o efluente gerado por uma indústria de cerveja. Ash e Truong (2003) avaliaram o potencial do capim Vetiver em Queensland no tratamento de esgoto na Austrália e observaram uma remoção de $85 \%$ do fósforo total.

\section{Nitrogênio Amoniacal}

As médias percentuais na redução de nitrogênio amoniacal foram de $95,3 \%$ no leito com solo; $86,3 \%$ nos leitos vegetados com $P$. bambusoides; $89,5 \%$ nos leitos com $P$. aurea e $98,2 \%$ no leito vegetado com $G$. angustifolia (Tabela 1 ). As eficiências na remoção do nitrogênio amoniacal foram bastante elevadas aos 60 dias do início da aplicação do esgoto nos leitos de tratamento. No entanto, reduziram drasticamente por volta dos 180 dias, permaneceram baixas até os 300 dias quando voltaram a crescer e ficaram todas acima de $90 \%$. O tratamento com G. angustifolia mais uma vez constituiu-se em exceção, mantendo uma eficiência acima de 95\%, desde o início da aplicação do esgoto (Figura 6).

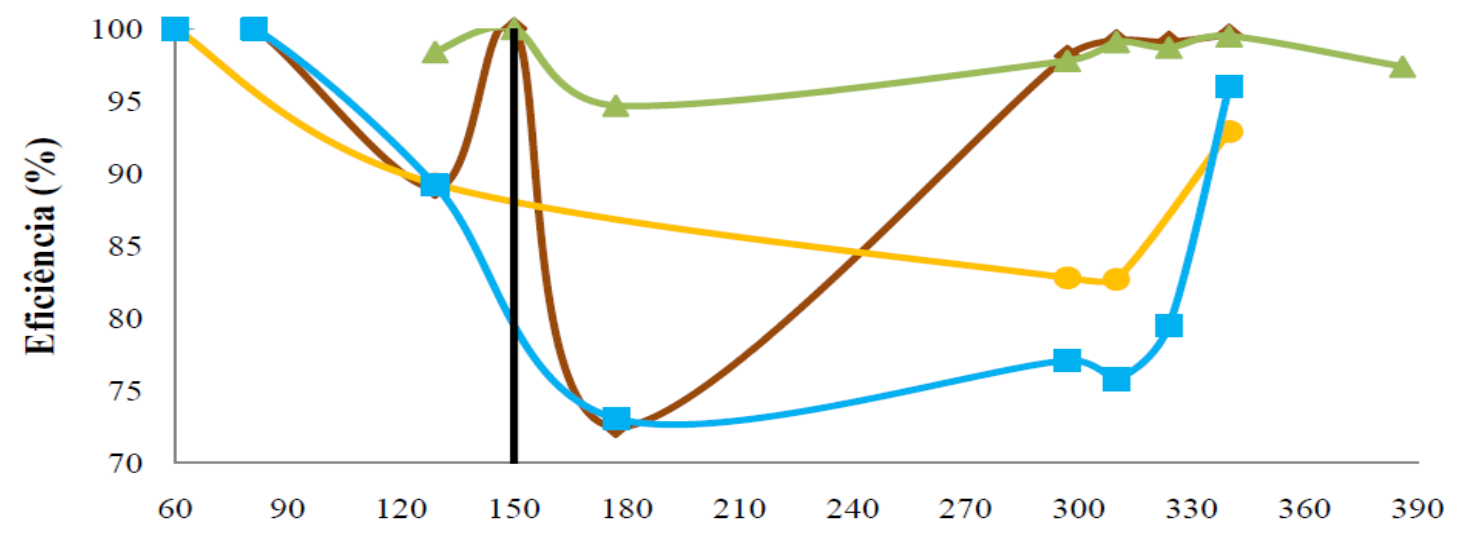

Período de aplicação de esgoto nos leitos após transplantio (dias)

$\multimap$ SOLO - ANGU $\multimap$ AUREA $\because$ BAMB - Mudança do TDH de 2,3 para 5,2 dias

Figura 6 Evolução da eficiência na remoção de nitrogênio amoniacal nos módulos de tratamento com bambu entre 60 a 390 dias após o início da aplicação do esgoto. Goiânia, GO. 2011.

A remoção eficiente depende principalmente da composição dos solos e do grau de escoamento superficial nos sistemas de alagados construídos (BRIX, 1987). Neste estudo verificouse remoção do nitrogênio com variação entre $86 \%$ e $98 \%$. No estudo de Pitaluga (2011) houve redução média percentual do nitrogênio amoniacal de $58,2 \%$ no sistema preenchido com areia; $58,3 \%$ no sistema preenchido com brita \# 0 e $59,5 \%$ no preenchido com substrato brita \# 1 , todos vegetados com a espécie Hedychium coronarium, em Goiânia, GO

Chagas (2008) obteve eficiência de 52,36\% na remoção de nitrogênio em sistemas de alagados construídos cultivados com lírio amarelo (Hemerocallis flava L.) na remoção de poluentes do efluente doméstico, em Viçosa, MG, com TDH de 3,9 dias. Almeida, Oliveira e Kliemann (2007) obtiveram num sistema de tratamento do tipo zona de raízes com fluxo subsuperficial descendente vegetado com lírio-do-brejo (Hedychium coronarium J. König) uma eficiência na redução do teor de nitrogênio amoniacal de 53,46\%, em Goiânia, GO. Ambos resultados inferiores ao obtido por este estudo (entre $86 \%$ e $98 \%$ ). 


\section{CONCLUSÕES}

A espécie Guadua angustifolia, de maneira geral, apresentou maior eficiência na remoção da carga poluidora de todos os atributos físico-químicos estudados.

Houve maior remoção da demanda bioquímica de oxigênio e de fosfato no TDH de 5,2 dias. Já o TDH de 2,3 dias mostrou-se mais eficiente para a remoção do nitrogênio amoniacal.

\section{REFERÊNCIAS}

ABRANTES, L. L. M. Tratamento de esgoto sanitário em sistemas alagados construídos utilizando Typha angustifolia e Phragmites australis. Dissertação (Mestrado em Engenharia do Meio Ambiente)-Escola de Engenharia Civil, Universidade Federal de Goiás, Goiânia, 2009. 140 p.

ALMEIDA, R. A.; OLIVEIRA, L. F. C.; KLIEMANN, H. J. Eficiência de espécies vegetais na purificação de esgoto sanitário. Pesquisa Agropecuária Tropical, v.37, n.1, p.1-9, 2007.

ALMEIDA, R. A.; UCKER, F. E. Considerando a Evapotranspiração no Cálculo de Eficiência de Estações de Tratamento de Esgoto com Plantas. Revista Engenharia Ambiental, Espírito Santo do Pinhal, v. 8, n. 4, p. 39-45. 2011.

APHA, Standard Methods for Examination of Water and Wastewater. 18² d, Washington, 1992.

ASH, R.; TRUONG, P. The use of vetiver grass for sewerage treatment. 2003. Disponível em: <http://www.vetiver.org/AUS_ekeshire01.pdf>. Acesso em: 14 dez. 2011.

BRIX, H. Treatment of wastewater in the rizhosphere of wetlands plants - the root zone method. Water Science and Technology, Great Britain, IAWQ, v. 19, n. 1, p. 107-118, 1987.

BRIX, H.; ARIAS, C. A.; JOHANSEN, N.H. Experiments in a two-stage constructed wetlands system: nitrification capacity and effects of recycling on nitrogen removal. In: VYMAZAL, J. (Ed). Wetlands - nutrients, metals and mass cycling. Leiden, The Netherlands: Backhuys Publishers, 2003. p. 237-258.

CHAGAS, R. C. Utilização de lírio amarelo (Hemerocallis flava) em sistemas alagados construídos para tratamento de esgoto doméstico.Dissertação (Mestrado em Engenharia Agrícola), Universidade Federal de Viçosa, Viçosa, 2008.66 p.

COSTA, L. L.; CEBALLOS, B. S. O.; MEIRA, C. M. B. S.; CAVALCANTI, M. L. F. Eficiência de wetlands construídos com dez dias de detenção hidráulica na remoção de colífagos e bacteriófagos. Revista biológica e ciências da terra, Paraíba, Editora da Universidade Estadual do Paraíba, v. 03, n. 01, 2003.

DINARDI, A. L.; FORMAGI, V. M.; CONEGLIAN, C. M. R.; BRITO, N. N.; DRAGONI, G.; TONSO, S.; PELEGRINI, R. Fitorremediação. In: III FÓRUM DE ESTUDOS CONTÁBEIS, Faculdades Integradas Claretianas, 2003, Rio Claro, SP.

FIGUEIREDO, C. C.; SANTOS, G. G.; PEREIRA, S.; NASCIMENTO, J. L.; ALVES JÚNIOR, J. Propriedades físico-hídricas em latossolo do cerrado sob diferentes sistemas de manejo. Revista Brasileira de Engenharia Agrícola e Ambiental, Campina Grande, v. 13, n. 02, mar. 2009. Disponível em: <http://www.scielo.br/scielo.php?pid=S141543662009000200006\&script=sci_artt ext>. Acesso em: 19 abr. 2010.

HABERL, R. Constructed wetlands: a chance to solve wastewater problems in developing countries. Water Science and Technology, v. 40, n. 3, p.1-17, 1999. 
Rev. Elet. em Gestão, Educação e Tecnologia Ambiental (e-ISSN: 2236-1170)

JASPER, S. P.; BIAGGIONI, M. A. M.; LOPES, A. B. C. Análise de custo de dois sistemas naturais de tratamento de água residuária na suinocultura. Energia na Agricultura, Botucatu, v.22, n.1, p.112-124, 2007.

MAYORCA, A. Vetiver system used in wastewater treatment. 2007. Disponível em: <http://www.vetiver.org/VEN_Beer_WW01pdf.pdf>. Acesso em: 12 dez. 2011.

MAZZOLA, M. Uso de leitos cultivados de fluxo vertical por batelada no pós-tratemento de efluente de reator anaeróbio compartimentado. Biblioteca digital da UNICAMP, Campinas, SP, 2003. Disponível em: $<$ http://libdigi.unicamp.br/document

/?code=vtls000299332>. Acesso em: 05 ago. 2009.

MEIRA, C.; CEBALLOS, B. S. O.; SOUZA, J. T.; KONIG, A. Wetlands vegetados no polimento de águas superficiais poluídas: primeiros resultados. In: XXI CONGRESSO BRASILEIRO DE ENGENHARIA SANITÁRIA E AMBIENTAL, 2001, João Pessoa. Anais... Rio de Janeiro: ABES, p. 1-6, 2001.

MOORTEL, A. M. K. V.; ROUSSEAU, D. P. L.; TACK, F. M. G.; PAUW, N. D. A comparative study of surface and subsurface flow constructed wetlands for treatment of combined sewer overflows: A greenhouse experiment. Ecological Engineering, v. 35, p. 175-183, 2009.

OLIVEIRA JÚNIOR, J. P.; CORRECHEL, V.; CUNHA, P. P.; LEANDRO, W. M.; GOGATO, G.; DEL'ACQUA, B. M. Efeito de palhadas de cobertura em atributos químicos do solo cultivado com algodoeiro em Goiânia, Goiás. In: CONGRESSO BRASILEIRO DE ALGODÃO, 5., 2005, Salvador, BA. Anais... Salvador, BA: EMBRAPA, 2005. Disponível em: <http://www.cnpa.embrapa.br/produtos/algodao/p

ublicacoes/trabalhos_cba5/410.pdf>. Acesso em: 19 abr. 2010.

PITALUGA, D. P. S. Avaliação de diferentes substratos no tratamento de esgoto sanitário por zona de raízes. Dissertação (Mestrado em Engenharia do Meio Ambiente)-Escola de Engenharia Civil, Universidade Federal de Goiás, Goiânia, 2011. 133 p.

SANTOS, H. G.; FIDALGO, E. C. C.; COELHO, M. R.; ÁGLIO, M. L. D. Cultivo do arroz de terras altas no estado de Mato Grosso. Embrapa arroz e feijão. Mato Grosso, v. 7, set. 2007. Disponível em: $<$ http://sistemasdeproducao.cnptia.embrapa.br /FontesHTML/Arroz/ArrozTerrasAltasMatoGrosso/solos.htm\#lvd>. Acesso em: 09 ago. 2011.

SOLANO, M. L.; SORIANO, P.; CIRIA, M. P. Constructed wetlands as a sustainable solution for wastewater treatment in small villages. Biosystems Engineering, v. 87, n. 01, p. 109-118, 2004.

SOUZA, L. E. L.; BERNARDES, R. S. Avaliação do desempenho de um RAFA no tratamento de esgotos domésticos, com pós-tratamento através de leitos cultivados. SIMPÓSIO ÍTALO-BRASILIANO DE INGENIERÍA SANITARIA-AMBIENTALE, 1., 1996, Gramado. Anais... Gramado: 1996.

TA-OUN, M.; THERAJINDAKAJORN, P.; PANCHABAN, S.; PRUANGKA, S. Vetiver grass research: primary management of wastewater from community. 2003. Disponível em: <http://www.vetiver.org/ICV3proceedings/THAl_wastewater\%

20treat.pdf>. Acesso em: 05 dez. de 2011.

VALENTIM, M. A. A. Uso de leitos cultivados no tratamento de tanque séptico modificado. Campinas, SP: 1999. Originalmente apresentada como dissertação de mestrado, Faculdade de Engenharia Agrícola - UNICAMP, 1999. 119 p.

ZANELLA, L. Plantas ornamentais no pós-tratamento de efluentes sanitários: Wetlands-construídos utilizando brita e bambu como suporte. Campinas, SP: Universidade Estadual de Campinas, 2008. Originalmente apresentada como tese de doutorado, Universidade Estadual de Campinas - UNICAMP, 2008. 219 p. 